\title{
VICTIMIZACIÓN SECUNDARIA EN EL PROCESO DE ENTREVISTA EN CÁMARA DE CIRCUITO CERRADO, CENTRO DE SERVICIOS AUXILIARES DE LA ADMINISTRACIÓN DE JUSTICIA DE FAMILIA
}

\section{SECONDARY VICTIMIZATION IN THE INTERVIEW PROCESS IN THE CLOSED CIRCUIT CHAMBER, AUXILIARY SERVICES CENTER OF THE FAMILY JUSTICE ADMINISTRATION}

Referencia del artículo

Nolasco Molina, I. J. (2021). Victimización secundaria en el proceso de entrevista en cámara de circuito cerrado, Centro de Servicios Auxiliares de la Administración de Justicia de Familia. Revista Científica del Sistema de Estudios de Postgrado de la Universidad de San Carlos de Guatemala, 4(1), 1-10. DOI: https://doi.org/10.36958/sep.v4i1.71

\section{Licda. Iris Janeth Nolasco Molina \\ inolasco@psicousac.edu.gt}

Departamento de Posgrados, Escuela de Ciencias Psicológicas, Maestría en Psicología Forense

Universidad de San Carlos de Guatemala

\section{RESUMEN}

Con el acuerdo 27-2014, de la Corte Suprema de Justicia se crea el "Centro de Servicios Auxiliares de la Administración de Justicia de Familia, alberga 8 Juzgados de Primera Instancia de Familia, mismos que requieren el auxilio del profesional en psicología, para que realice la entrevista en cámara de circuito cerrado de televisión. Para desarrollar la entrevista el profesional en psicología realiza su función con lineamientos análogos, porque aún se carece de un protocolo específico de atención a la niñez y adolescencia en el ámbito jurídico familiar; condición que algunas veces limita la atención idónea.

La investigación permitió identificar cómo es la atención que reciben las personas encargadas o padres de familia que traen a una persona para que se le realice la entrevista; se implementó con el enfoque cuantitativo, y modelo descriptivo, utilizando un cuestionario aplicado a la muestra.

Se realizó el análisis estadístico descriptivo, los resultados establecen que las personas que traen a entrevista a niños y adolescentes desconocen el procedimiento a seguir, generándose la victimización secundaria. Al presentar los resultados de la investigación a la magistrada Silvia Molina, se inició el proceso de elaboración del Protocolo de atención a NNA y personas con discapacidad por parte del equipo multidisciplinario de los Juzgados de Primera Instancia de Familia.

\section{PALABRAS CLAVE}

Victimización secundaria, padres o encargados, niñez, adolescencia, entrevista, Cámara Circuito Cerrado, protocolo

\section{ABSTRACT}

With the agreement 27-2014, the Supreme Court of Justice creates the "Auxiliary Services Center of the Administration of Family Justice, it houses 8 Courts of First Instance of Family, which require the help of the professional in psychology, to conduct the interview on a closed circuit television camera. To develop the interview, the professional in psychology performs his function with similar guidelines, because there is still no specific protocol of attention to children and adolescents in the family legal field; a condition that sometimes limits the ideal care.

The research made it possible to identify how the care received by the persons in charge or parents who bring a person to be interviewed; It was implemented with a quantitative approach and a descriptive model, using a questionnaire applied to the sample.

Descriptive statistical analysis was carried out, the results establish that the people who bring children and adolescents to the interview do not know the procedure to follow, generating secondary victimization. Upon presenting the results of the investigation to Judge Silvia Molina, the process of elaboration of the Protocol of attention to NNA and people with disabilities began by the multidisciplinary team of the Family Courts of First Instance.

\section{KEYWORDS}

Secondary victimization, parents or guardians, childhood, adolescence, interview, Closed Circuit Chamber, protocol 


\section{INTRODUCCIÓN}

El presente artículo es derivado de la investigación denominada "Proceso de Atención a NNA/personas adultas con discapacidad en la entrevista de Cámara de Circuito Cerrado" (Nolasco 2019). En este se evidencian los datos arrojados por la evaluación realizada en el año 2,019 a las personas que asisten a los niños, niñas, adolescentes a la entrevista en cámara de circuito cerrado, en los juzgados de conocimiento del centro de justicia de familia. La misma se realizó para establecer la existencia de revictimización en el proceso, ante la falta de un protocolo específico de atención, que indique el funcionamiento del equipo multidisciplinario, para evitar vulnerar a la persona que se atiende.

Existen principios normativos que rigen la labor de los funcionarios de los juzgados de familia, mismos que preceden estándares internacionales que contemplan la protección de los derechos humanos como: la declaración universal de los derechos humanos, las reglas de Brasilia, que tienen como principio "atender a la persona que se encuentra en estado de vulnerabilidad sin discriminación alguna", y que la persona que participe en un proceso judicial debe ser atendida en una sala adecuada, facilitarle la comprensión del procedimiento. (XIV Cumbre Judicial Iberoamericana, 2008) numerales $(1,40,41,78)$ La convención de los derechos del niño en 1,998 en el artículo 3 que en "todas las medidas que tomen las instituciones públicas o privadas se debe atender el interés superior del niño". (COPREDEH, 2011)

También los protocolos de entrevista en cámara de Gesell, mismo que ha sido ratificado por la Corte Suprema de Justicia según Acuerdo 16-2013, en el mismo se describen el instructivo para el uso y funcionamiento de la cámara de Gesell, Circuito Cerrado, para que los auxiliares judiciales implemen- ten. Los profesionales de psicología deben apegarse a lo establecido en el Código de ética (Guatemala, 2008-2010). Es valida esta investigación, toda vez que se logró conocer la opinión que tienen los usuarios, en relación a la atención que reciben cuando asisten a una entrevista en la cámara de circuito cerrado.

De tal manera que se pudo evidenciar a través de información objetiva veraz y con el rigor científico, si se cumple con los principios de celeridad y calidad las funciones asignadas, para satisfacer las necesidades de la persona usuaria, garantizando sus derechos fundamentales, así mismo el "actuar con integridad responsabilidad, prudencia, respeto, eficiencia, eficacia, efectividad" en pro de la dignidad de la persona. (Organismo Judicial , 2013)

La investigación aborda como tema central el análisis de variables de victimización secundaria en el proceso de entrevista en la cámara de circuito cerrado, incluyendo las variables de atención antes, durante y después de la entrevista, las condiciones del entorno físico y el actuar del personal en relación a los principios éticos.

El entorno físico incluye variables como: falta de equipamiento en la sala de espera, guardería y sala de entrevista, hacinamiento en la sala de guardería y distractores en la sala de entrevista. A la categoría de personal de apoyo le asisten las variables: desconocimiento de procedimientos, falta de personal y a las pautas del entrevistador las variables implicadas son: pautas de la entrevista, principios éticos.

\section{MÉTODOS Y MATERIALES}

Se utilizó el enfoque cuantitativo, fundamentado desde el modelo exploratorio des- 
criptivo, no experimental, que se evidencia a través del marco teórico desarrollado y la descripción de la información que dieron los participantes. Se utilizó una muestra no aleatoria, de 7 personas, entre hombres y mujeres, en edades comprendidas entre los 25 a 70 años, padres o encargados de los NNA, o persona con discapacidad que asisten a la entrevista en cámara de circuito cerrado.

El cuestionario para padres o encargados se conformó de 15 preguntas, se utilizaron las variables de entorno físico, pautas del entrevistador, antes durante y después de la en- trevista, así también el accionar del personal de apoyo (oficial y auxiliar de guardería).

La técnica de análisis de datos fue factorial, se desarrollaron gráficas de las variables homogéneas, procurando la independencia entre ellos. Esto llevó a encontrar grupos de respuesta común, para reducir el número de dimensiones necesarias de los datos. Con el programa estadístico SPSS, se tabularon los datos y se agruparon a través de gráficos de barra, describiendo los resultados.

\section{RESULTADOS}

Figura 1. Acompañamiento del oficial y profesional de psicología antes de la audiencia.

\section{Acompañamiento del oficial y profesional de psicología antes de la audiencia}

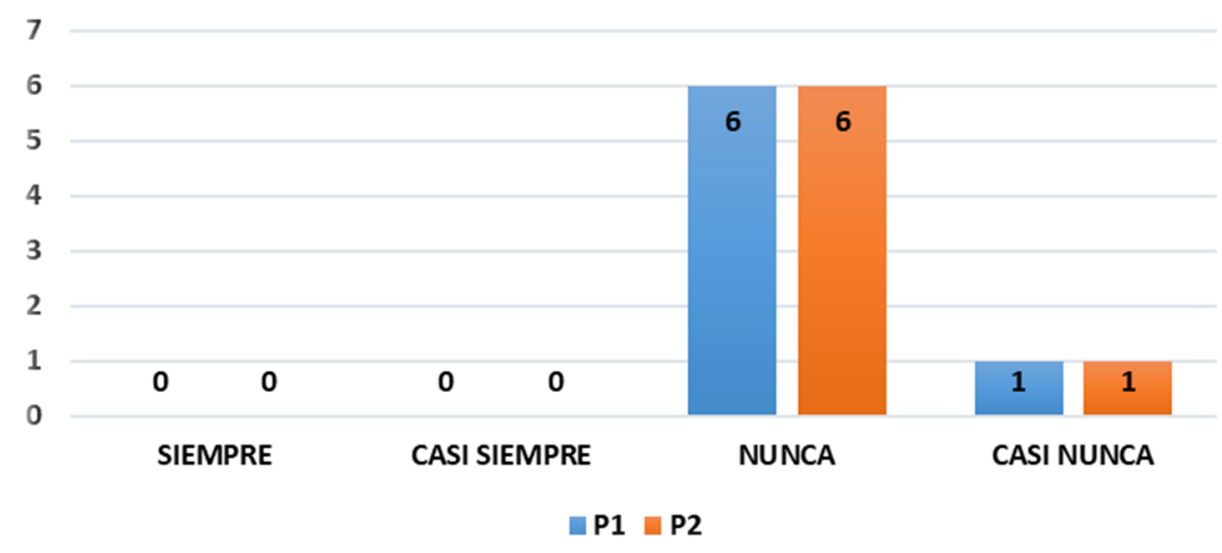

Fuente: Elaboración propia. Encuesta realizada a padres o encargados que asisten al centro de Servicios Auxiliares de la administración de justicia de familia.

La gráfica 1 representa según la encuesta realizada a padres y encargados, que asisten al centro de servicios auxiliares de justicia de familia, con las preguntas de investigación: 1. ¿Antes de la audiencia el oficial le da instrucciones específicas del procedimiento a realizarse? 2. ¿Antes de la entrevista el profesional de la psicología le da instrucción del procedimiento a realizarse? 
Dando como resultado que de los 7 participantes, 6 manifiestan que nunca y 1 que casi nunca, el oficial, y el profesional en psicología, les da instrucciones específicas del procedimiento a realizarse antes de la audiencia. Esto les crea confusión, incertidumbre, ante la falta de ilustración, que les permita seguir un procedimiento estandarizado, y les guíe adecuadamente, cuando se va a llevar a cabo una entrevista en cámara de circuito cerrado de televisión.

Figura 2. Acompañamiento del personal de guardería antes, durante y después de la audiencia.

\section{Acompañamiento del personal de guarderia antes, durante y después de la audiencia}

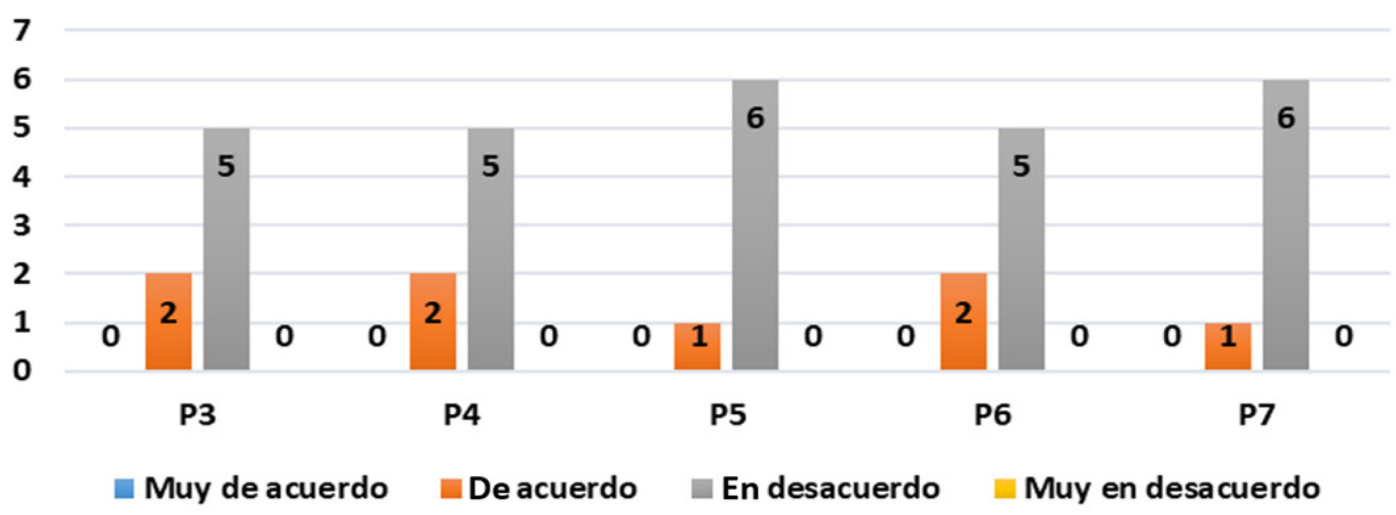

Fuente: Elaboración propia. Encuesta realizada a padres o encargados que asisten al centro de Servicios Auxiliares de la administración de justicia de familia.

La gráfica 2, evidencia los resultados de las preguntas: 3 ¿Cuándo llega al juzgado para audiencia de entrevista en cámara de circuito cerrado, lleva al NNA o persona con discapacidad, al área de guardería? 4 ¿Las personas de área de guardería prestan atención en espacios adecuados a las necesidades y material para cubrir alguna emergencia?, 5 ¿Les lleva a la sala de entrevista? 6 ¿El personal que atiende es suficiente? 7 ¿El personal de guardería cuenta con materiales necesarios para cubrir alguna emergencia de NNA / persona con discapacidad?.
La misma representa la percepción que tienen los evaluados, con relación al acompañamiento que brinda la persona auxiliar del área de guardería; a las preguntas 3,4, y 6 indican estar en desacuerdo, indicando que al llegar al juzgado para la audiencia de entrevista en cámara de circuito cerrado, llevan al NNA/persona con discapacidad, al área de guardería, las personas de área de guardería prestan atención en espacios inadecuados a sus necesidades, que no es suficiente dicho personal para atenderlos según edad. Dos personas indicaron que están de acuerdo con los cuestionamientos formulados. A la pregunta 5 y 7,6 respondieron que están en des- 
acuerdo porque no llevan a los NNA / persona con discapacidad a la sala de entrevista, y no cuentan con materiales necesarios para cubrir alguna emergencia; es decir que la ma- yoría de los padres y encargados consideran que hay deficiencias en la atención que reciben en el área de guardería, o en su mayoría desconocen que existe una guardería.

Figura 3. Condiciones del espacio físico

\section{Condiciones del espacio físico}

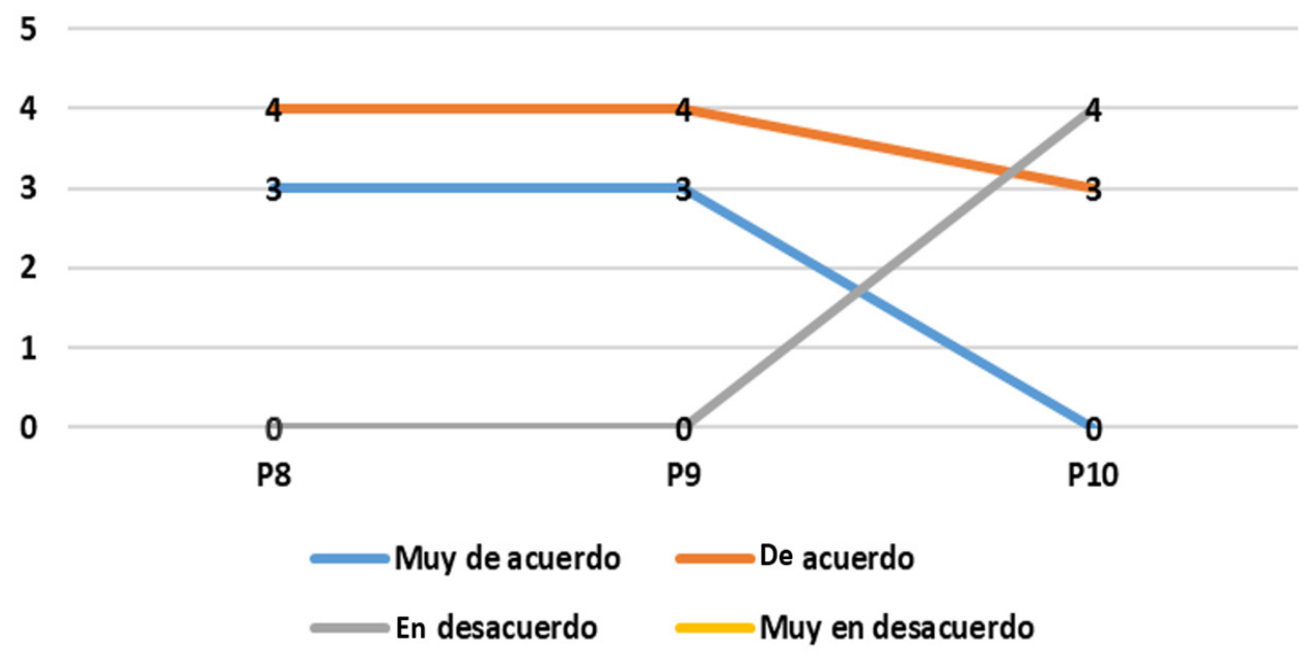

Fuente: Elaboración propia, Encuesta realizada a padres o encargados que asisten al centro de justicia de familia.

La gráfica 3, responde a los datos recabados de las respuestas a las interrogantes: 8 . ¿Existen distractores de la armonía y tranquilidad en sala de guardería? 9. ¿Le ofrecen agua, u otro insumo básico mientras espera? 10. ¿El área de espera es cálida, confortable, con música relajante?

Se describe que a la pregunta número 8 y 9, 3 personas están muy de acuerdo y 4 de acuerdo, con respecto a que existen distractores de la armonía y tranquilidad en sala de guardería, el área de espera no es cálida, ni confortable, carece de música relajante; al mismo tiempo que el personal de guardería, no les ofrecen agua $u$ otro insumo mientras esperan. A la pregunta 10, 3 encuestados están de acuerdo, consideran que la sala de espera es cálida confortable y con música relajante, esto está relacionado con la atención que se le brinda en una de las salas de atención para la entrevista, si se cuenta con las variables indicadas. Los 4 restantes expresaron estar en desacuerdo con las premisas. 
Figura 4. Pautas en proceso de entrevista del Profesional en psicología

\section{Pautas en proceso de entrevista del Profesional en psicología}

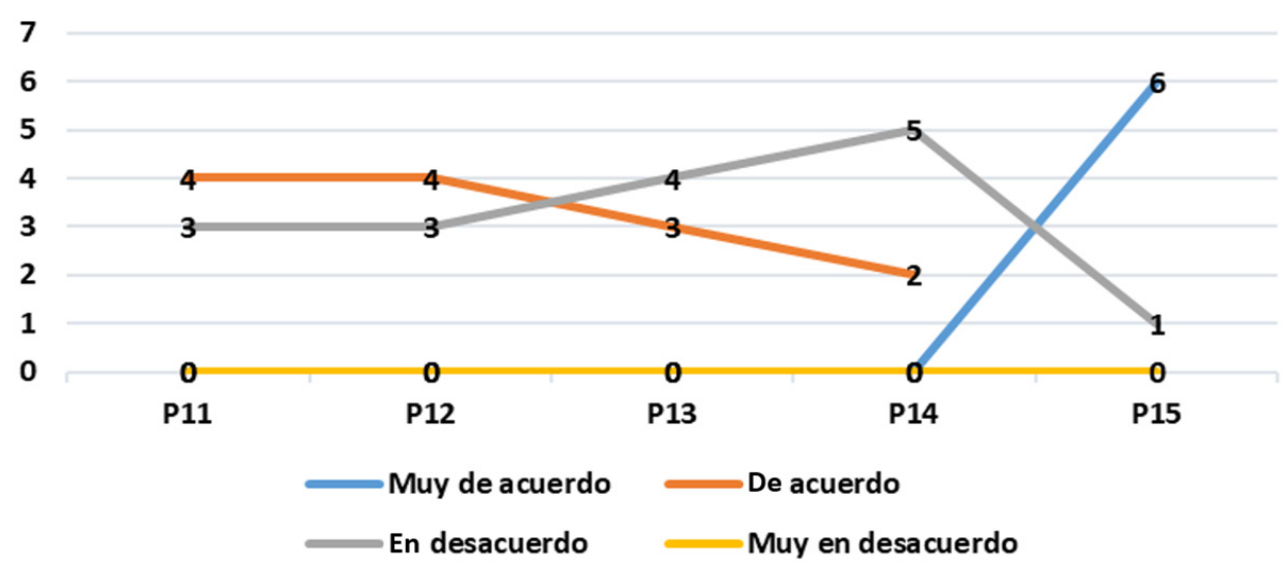

Fuente: Elaboración propia, Encuesta realizada a padres o encargados que asisten al centro de justicia de familia.

La gráfica 4, evidencia los resultados a las preguntas: 11. ¿El profesional de la psicología, 15 minutos antes de la entrevista con el juez, inicia su labor con el NNA / persona con discapacidad? 12. ¿El profesional de la psicología explica el procedimiento al encargado del NNA o persona con discapacidad, firma consentimiento informado? 13. ¿Estuvo presente en el desarrollo de la entrevista? 14. ¿El profesional de la psicología le dan atención para bajar la tensión (ejercicios de respiración) previo a que deje al NNA/ persona con discapacidad, para la entrevista. 15. ¿Demuestra respeto e interés a atenderle?

Obteniendo 5 respuestas de muy en desacuerdo a las preguntas de la 11 a la $14, y$ la pregunta 15 , solo dos personas están muy en desacuerdo. A las preguntas 11,12 , hay 4 respuestas de acuerdo y 3 en desacuerdo, es decir que la mayoría coinciden con estar de acuerdo en que el profesional de la psicología, les recibe 15 minutos antes, explica el procedimiento y firma consentimiento infor- mado. A la pregunta 13, hay 3 respuestas de acuerdo y 4 en desacuerdo, es decir que la minoría indicaron estar de acuerdo porque se les permitió por parte del juez estar presente en la entrevista. En la pregunta 14 la mayoría describió estar en desacuerdo ante la afirmación, a recibir atención para bajar la tensión (ejercicios de respiración) previo a que deje al NNA/ persona con discapacidad, para la entrevista, tampoco les ofrecen agua u otro insumo básico mientras esperan. Para la pregunta 15, 6 de los entrevistados indicó estar de acuerdo, y 1 en desacuerdo, es decir que la mayoría está de acuerdo en que recibe las muestras de respeto e interés al atenderles por parte del profesional en psicología.

\section{DISCUSIÓN DE RESULTADOS}

Esta investigación se realizó gracias a las personas que aceptaron ser parte de la muestra, quienes asistieron a la entrevista en Cámara 
de Circuito Cerrado al Centro de Servicios Auxiliares de Familia.

En el protocolo de atención integral para la víctima del delito del Ministerio público (MP2014) se establece que según las observaciones de Naciones Unidas (1985) la "victimización secundaria", es aquella victimización que ocurre a través de la respuesta de las instituciones y las personas, hacia quienes asisten a resolver su problemática, en este caso un conflicto del ámbito familiar.

La encuesta ha revelado información fundamental en relación con la atención dentro del Centro de Justicia de Familia. Según los padres y encargados de los niños y adolescentes que asisten a entrevista, en su mayoría describió que el personal responsable de brindar atención idónea cumple de manera parcial con atender a las personas. Brindando escuetamente las instrucciones al usuario, para que siga una ruta de acceso que lo lleve a sentirse seguro.

El acompañamiento de la auxiliar de guardería es insuficiente, dado el poco personal, por lo tanto una persona no puede ir a otro nivel del edificio a dejar un niño a la sala de entrevista, si tiene más niños a su cargo; por otro lado, los padres o encargados muchas veces desconocen que hay una guardería y comó funciona. Las personas que han llegado al área de guardería se percatan que los espacios son hacinados, falta de tranquilidad y armonía, los pasillos siempre están confinados de abogados y personas que vienen a los juzgados.

Los resultados evidencian que la minoría de personas que asiste a un NNA o persona con discapacidad, para que sea entrevistado, han vivenciado que los profesionales en psicología, les atienden 15 minutos antes de la entrevista con el juez, para el desarrollo del espacio de confianza, les explican el proce- dimiento a realizar, y firma consentimiento informado, les dan atención para bajar la tensión (ejercicios de respiración) previo a que deje al NNA/ persona con discapacidad, para la entrevista. No obstante la mayoría manifestó que les demuestran respeto e interés al atenderle.

Cabe mencionar que los profesionales en psicología, tienen una sala de entrevista que comparten como sala de evaluación, con diferentes distractores, como teléfono, ruido externo, mesas y sillas inadecuadas.

Otro dato importante es el resultado que evidencia que algunos padres o encargados de los entrevistados ingresan a la sala de audiencia a presenciar la entrevista, la mediación del juez dirigiendo la entrevista, condiciones que se dan por falta de procedimiento oportuno, que permita cumplir con los estándares y tratados internacionales, en procura de respetar los derechos humanos.

En general el resultado de la encuesta evidencia la falta de procedimientos estandarizados, espacios adecuados y poco personal en el área de guardería; por lo tanto la confusión y desorientación, que puede vivenciar un padre o encargado, al traer a una persona a que se le realice la entrevista en cámara de Circuito Cerrado, desarrolle la victimización secundaria. Con respecto a los profesionales de la psicología, aún se requiere que todos desarrollen los protocolos para evitar la victimización secundaria.

Es decir que el "instructivo para el uso y funcionamiento de la cámara..." aprobado a través del acuerdo número 16-2013 por la corte Suprema de Justicia, sea llevado a la práctica en su totalidad. Organismo Judicial (2013). 


\section{CONCLUSIONES}

La participación del sistema de justicia es fundamental para asegurar la aplicación de la justicia, visibilizada a través de la atención y protección que se brinda; si los actores de un juicio reciben un acompañamiento adecuado, que implique el respeto a su dignidad y demás derechos que le asisten, podrán confiar en el sistema y en sí mismo, y se evitará la victimización secundaria.

En el Centro de Justicia de Familia existen 6 salas de cámara de circuito cerrado, y solo hay una persona encargada de dar cuidado en guardería, este es uno de los aspectos de alta incidencia de victimización y disminución en la atención óptima. Es imposible atender a los diferentes grupos de edades que asisten, no tienen agenda para saber cuándo hay audiencia, el espacio físico es muy reducido, carecen de un sanitario apropiado para los entrevistados.

Los oficiales en su mayoría tienen recarga excesiva de trabajo, audiencias, eso limita que estén anuentes a informar a los usuarios el procedimiento para que se realice la entrevista. Sin embargo, si se coordinan con los psicólogos para desarrollar la entrevista, previo a que el juez inicie la misma. No así con el juez, quien carece de tiempo para organizar y plantear sus inquietudes al profesional en psicología previo a la audiencia. Existen juzgadores que permiten que los padres, o encargados ingresen a la sala de audiencia, provocando revictimización porque cuando se llevan a los niños los cuestionan en cuanto a las respuestas que brindaron durante la entrevista.

El profesional de psicología desarrolla el procedimiento oportuno de las pautas de la entrevista, en relación con la atención previa, las instrucciones de procedimiento, el trato con respeto, no obstante con distractores para que desarrolle su función profesional en óptimas condiciones. Algunos carecen de un espacio físico pertinente, mobiliario específico para la entrevista, ya que comparte espacio para evaluar a los usuarios adultos, tiene interrupciones de otros usuarios o llamadas telefónicas, color de las paredes no es el establecido en la guía, mantienen imágenes en las paredes, libreras con libros y objetos.

Al terminar la investigación se dieron a conocer los resultados a la dirección de familia y gracias a la anuencia a mejorar y cumplir con los principios del organismo Judicial, la Licda. Karla Mancio, Coordinadora de la Dirección de Familia y a requerimiento de la Señora Magistrada Dra. Silvia Verónica García Molina Vocal VIII de la Corte Suprema de Justicia, se instauró la mesa Técnica para la elaboración del documento "Protocolo de atención a Niñas, Niños y Adolescentes y Personas Adultas con Discapacidad" el mismo continúa en espera de dictamen favorable desde marzo de 2020.

\section{RECOMENDACIONES}

La dirección del Centro de Justicia de Familia debe dar seguimiento a la mesa técnica instaurada, para que se apruebe y publique el "Protocolo de atención a Niñas, Niños y Adolescentes y Personas Adultas con Discapacidad".

Para el desarrollo eficiente del proceso de entrevista, el equipo multidisciplinario (auxiliar de guardería, oficial, juez, psicólogo, padre o encargado) debe conocer el protocolo de atención y dar seguimiento al mismo para evitar la victimización secundaria. Se debe contar con el número de personal que logre atender eficientemente a las personas usuarias del sistema de justicia de familia. Se deben condicionar los espacios físicos, 
acorde a las necesidades de la población que se atiende y contar con los implementos necesarios.

\section{REFERENCIAS}

COPREDEH. (5 de 11 de 2011). www.copredeh.gob.gt. Obtenido de Concencion Internacional sobre los derechos del niño: http://www.copredeh.or.cr

Guatemala, C. d. (octubre de 2008-2010). Código de Ética. Colegio de Psicólogos de Guatemala, "Código de Ética", Tribunal de Honor. Guatemala, Guatemala, Guatemala.

M.P. Introducción 04-2014, Protocolo atención Integral para Víctimas del delito. http:/ /www.mp.gob.gt

Organismo Judicial. (2013). Corte Suprema de Justicia. Acuerdo 22-2013. Guatemala, Guatemala: Departamento de Comunicación Social.

Organismo Judicial. (2013). Acuerdo 162013. Instructivo para el uso y funcionamiento de la Cámara de Gesell, Circuito Cerrado y otras herramientas para recibir las declaraciones de niños, niñas y adolescentes víctimas y /o testigos. Guatemala, Guatemala: CENADOJ.

XIV Cumbre Judicial Iberoamericana. (16 de octubre de 2008). 100 normas de Brasilia sobre acceso a la justicia de las personas en condición de vulnerabilidad. Obtenido de https/www.acnur.org

Nolasco Molina, Iris Janeth (2019) Proceso de Atención a NNA/personas adultas con discapacidad en la entrevista de Cámara de Circuito Cerrado.

100 Reglas de Brasilia (2018) acceso a la justicia de las personas en condición de vulnerabilidad.

\section{SOBRE AUTORA}

\section{Licda. Iris Janeth Nolasco Molina}

Es Psicóloga, de la Escuela de Ciencias Psicológicas de la USAC, Psicóloga Forense por la Universidad de San Carlos de Guatemala, Master en Resolución de Conflictos, y Mediación por la Universidad de Cervantes de España, Profesora revisora de procesos de investigación con fines de graduación de la Escuela de Ciencias Psicológicas de la USAC, Psicóloga en los Juzgados de Familia del Centro de Servicios Auxiliares de Familia, Organismo Judicial. Docente de la maestría en psicología Forense de la Escuela de Ciencias Psicológicas. 


\section{Conflicto de intereses \\ Declara no tener ningún conflicto de intereses.}

Declaración de consentimiento informado

El estudio se realizó, respetando el Código de ética y buenas prácticas editoriales de publicación.

\section{Derechos de uso}

Copyright $\odot 2021$ por Iris Janeth Nolasco Molina

Este texto está protegido por una licencia Creative Commons 4.0. Internacional

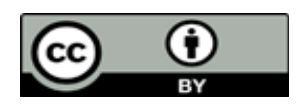

Usted es libre para compartir, copiar y redistribuir el material en cualquier medio o formato y adaptar el documento, remezclar, transformar y crear a partir del material para cualquier propósito, incluso comercialmente, siempre que cumpla la condición de atribución: usted debe reconocer el crédito de una obra de manera adecuada, proporcionar un enlace a la licencia, e indicar si se han realizado cambios. Puede hacerlo en cualquier forma razonable, pero no de forma tal que sugiera que tiene el apoyo del licenciante o lo recibe por el uso que hace. 